\title{
PCSK9-inhibition in routine clinical practice: real world data from Germany
}

\author{
E. Waldmann, J. Altenhofer, K. Henze, K.G. Parhofer
}

\section{Introduction}

PCSK9-inhibitors (PCSK9-i) are a new treatment option for patients not reaching their LDL-cholesterol targets. Efficacy and safety is good in study settings, but there is only few data on real world experience. We analyzed data from 146 consecutive patients who received PCSK9-i and participated in an observational study (lipid clinic; monocentric).

\section{Methods}

Lipid profiles were obtained (age $63 \pm 10$ y, BMI $23.6 \pm 4.1$ $\mathrm{kg} / \mathrm{m} 2,49 \%$ male) before and after 3-4 months treatment with PCSK9-I.

\begin{tabular}{lcrrc}
\hline & $\begin{array}{c}\text { baseline } \\
(\mathbf{m g} / \mathbf{d l})\end{array}$ & $\begin{array}{c}\text { after } \mathbf{3} \text { months } \\
(\mathbf{m g} / \mathbf{d l})\end{array}$ & $\begin{array}{c}\text { change } \\
(\%)\end{array}$ & p-value \\
\hline cholesterol & $250.3 \pm 72.1$ & $161.5 \pm 51.7$ & $-34.4 \pm 14.5$ & $<0.001$ \\
triglycerides & $168.5 \pm 102.8$ & $163.0 \pm 99.5$ & $4.1 \pm 42.5$ & 0.440 \\
HDL-cholesterol & $55.9 \pm 15.4$ & $55.5 \pm 15.3$ & $-0.2 \pm 11.0$ & 0.378 \\
Non-HDL-cholesterol & $194.4 \pm 70.4$ & $106.0 \pm 49.4$ & $-44.7 \pm 18.0$ & $<0.001$ \\
LDL-cholesterol & $161.0 \pm 58.9$ & $80.1 \pm 42.7$ & $-49.9 \pm 18.8$ & $<0.001$ \\
VLDL-cholesterol & $33.7 \pm 42.0$ & $26.0 \pm 27.4$ & $1.9 \pm 87.1$ & 0.001 \\
VLDL-triglycerides & $145.9 \pm 104.7$ & $143.1 \pm 99.7$ & $8.7 \pm 58.9$ & 0.499 \\
lipoprotein(a) & $48.6 \pm 48.5$ & $39.8 \pm 40.6$ & $-24.2 \pm 20.4$ & $<0.001$ \\
\hline
\end{tabular}

Fig.1: Parameters of lipid metabolism before and under 3 months PCSK-9-i therapy in $\mathrm{mg} / \mathrm{dl}$

\begin{tabular}{lccc}
\hline & $\begin{array}{c}\text { baseline } \\
(\mathrm{mg} / \mathrm{dl})\end{array}$ & $\begin{array}{c}\text { after 3 months } \\
(\mathrm{mg} / \mathrm{dl})\end{array}$ & $\begin{array}{c}\text { change } \\
(\%)\end{array}$ \\
\hline Alirocumab & & & \\
LDL-cholesterol & $158.2 \pm 55.6$ & $80.6 \pm 42.7$ & $-48 \% \pm 19$ \\
lipoprotein(a) & $52.2 \pm 57.5$ & $43.7 \pm 46.6$ & $-23 \% \pm 23$ \\
Evolocumab & & & \\
LDL-cholesterol & $174.7 \pm 80.1$ & $89.6 \pm 67.1$ & $-50 \% \pm 21$ \\
lipoprotein(a) & $46.8 \pm 63.7$ & $41.6 \pm 58.5$ & $-24 \% \pm 25$ \\
\hline
\end{tabular}

Fig.2: Parameters of lipid metabolism comparing Evolocumb and Alirocumab in $\mathrm{mg} / \mathrm{dl}$

\section{Results}

44\% received Evolocumab 140mg and 56\% Alirocumab (29\% 75mg and 27\% 150mg) q 2 weeks. Patients had either (95\%) atherosclerotic disease or familial hypercholesterolemia without atherosclerosis (5\%). All were on maximally tolerated lipid lowering therapy. Statin intolerance was prominent (complete 44\%, partial 25\%). Baseline LDL-C was reduced by 50\% (Fig.1); there was no significant difference between Alirocumab and Evolocumab (Fig.2). 17 (12\%) patients had a LDL-c reduction of $<30 \%$. In 8 lipid lowering therapy was reduced, in $4(2.7 \%)$ no reason was detectable, 3 varied considerably with stronger reductions at earlier or later time points. In 2 injection was longer than 2 weeks ago. Treatment was tolerated well. No patient stopped PCSK9-i within the first 3 months.

\section{Conclusions}

Alirocumab and Evolocumab reduce lipids safely in routine clinical practice. Statin intolerance is one of the main reasons to initiate PCSK9-inhibitors. True non-responders are rare $(<3 \%)$. Baseline LDL-C is considerably higher than in outcome studies $(161 \mathrm{mg} / \mathrm{dl}$ vs. $90 \mathrm{mg} / \mathrm{dl})$. Whether this translates into even greater clinical benefit than in the studies remains to be evaluated. 